\title{
Communiquer les connaissances: des pistes pour la mise en scène des sciences
}

Jacques Sapiega, Chercheur au LESA, enseignant au Département SATIS de l'Université de Provence

Rémi Adjiman, Chercheur à I'IRSIC, enseignant au Département SATIS de l'Université de Provence

Or, la question de la communication se pose désormais dans la (quasi) totalité des laboratoires de recherche, du moins au CNRS

La notion de grand public à laquelle il est fréquemment fait recours en matière de médiation scientifique mérite que l'on s'y arrête quelque peu. Dans le champ de la communication, les publicitaires ne s'y trompent pas lorsqu'ils mènent des enquêtes d'opinion auprès du public visé par le produit qu'ils sont chargés de promouvoir, la connaissance du public visé étant indispensable, le résultat des ventes y étant étroitement associé. Dans le champ de la médiation scientifique, cette notion de grand public peut être un fourre tout, voire une représentation uniforme du public, alors que des distinctions sont opérées lorsqu'il s'agit de publics scolaires (on ne s'adresse pas de la même manière à un enfant de classe maternelle, à un jeune de dix ans et à un lycéen ; on peut encore affiner la connaissance de ces publics et prendre en compte d'autres caractéristiques telles que, par exemple, l'environnement culturel de l'enfant ou encore la filière suivie par les lycéens). A contrario, dès que l'on

Depuis plus de dix ans nous produisons et fabriquons, dans un contexte pédagogique ${ }^{1}$, des films et contenus audiovisuels scientifiques.

Du point de vue de la réalisation, chacun des projets est bien unique et spécifique, souvent atypique même, et répondant à des contraintes à chaque

\footnotetext{
1 Au sein du Département SATIS (Sciences Arts et Techniques de I'Image et du Son) de I'Université de Provence. Cette formation délivre un Master à de futurs professionnels de l'audiovisuel dans les domaines du montage, de la prise de vue, du son à l'image, de la production-réalisation et de la musique pour l'image.
} 
fois particulières. Cela conduit à des réalisations totalement différentes, tant dans leur approche que dans leur durée, tant dans leurs objectifs, leurs destinataires, que dans leur genre. Parfois documentaires, parfois fictions, parfois multimédia, parfois installation, ces projets nous ont conduit à faire nous-mêmes de nombreux apprentissages. Un cadre de production a, à chaque fois, été envisagé, parfois avec un financement limité et très précaire obtenu alors que le projet était déjà bien lancé avec les étudiants, parfois avec davantage de moyens financiers obtenus en amont.

$\mathrm{Au}$ cours du temps, les méthodologies de réalisation et les approches pédagogiques se sont affinées, des modèles d'accompagnement ont été élaborés, des groupes d'experts ont été associés... Analyse et réalisation, réflexion et action sont toujours allés de pair dans cet ensemble. Une " pragmatique » s'est mise en place, portée non par des par des individus isolés mais par un ensemble de projets et d'engagements dictés par des opportunités stimulantes.

On peut ainsi brièvement citer des réalisations comme le DVD « La Durance » réalisé en 2002-2004, une installation pour l'" année mondiale de la physique » en 2004-2005, le dispositif Darius Milhaud² fait en 2006-2007, le DVD Aix-Marseille Universités en 2006-2007, une installation, exposition sur le Corail rouge en Méditerranée 2007-2008, un DVD pour Thalès technologies en 2007-2008 et un DVD sur le thème "La vie ailleurs » en $2008,2009 \ldots{ }^{3}$

Pour ce travail de recherche, nous ne prétendons pas avoir - a priori - été totalement rigoureux dans la sélection d'un corpus parfaitement représentatif des différentes formes de communication audiovisuelle scientifique ${ }^{4}$.

\footnotetext{
${ }^{2}$ Darius Milhaud, compositeur français et adepte de l'écriture polytonal déclarait : "Je suis méditerranéen, mais d'une Méditerranée allant de Constantinople à Buenos-Aires dont Aix en Provence est la capitale !»

${ }^{3}$ Le DVD « la Durance » est un projet commandé par la Régie Culturelle Régionale PACA, l'installation Mécaniques relatives, dans le cadre de l'« année mondiale de la physique " "était une commande de la Société Française de Physique, la Région PACA, le CG13 et la Ville d'Aubagne, le dispositif Darius Milhaud était une volonté de I'ARCADE et la Région PACA, le DVD Aix-Marseille Universités était une commande des 3 universités d'AixMarseille, l'installation exposition sur le Corail Rouge est une demande du laboratoire Dimar, les archives municipales de Marseille, Zinc et la Régie Culturelle Régionale, la commande du DVD Thalès technologies émane de Thalès, le projet " la vie ailleurs " provient d'un souhait de l'OAMP et de la Régie Culturelle Régionale...
}

${ }^{4}$ Comme a pu le faire Igor Babou lorsque, dans l'article qu'il écrit dans les actes du XIle 
Pourtant, nous pouvons noter que chacune de ses œuvres - si elles s'adressent globalement au grand public - cible à chaque fois des publics différents comme les collégiens, les lycéens, les amateurs de musique, les partenaires institutionnels de l'Université... Comme le précise Doazan, Damoiseaux et Botti dans leur article Passeurs de science (dans ce même ouvrage), "Dans le champ de la médiation scientifique, cette notion de grand public peut être un fourre tout, voire une représentation uniforme du public, alors que des distinctions sont à opérer ».

De plus, ces films nous permettent - dans la diversité de leurs approches, de leur durée, de leur forme, de leur commanditaire - de rendre compte des différentes expériences rencontrées, de les confronter à l'évolution de nos observations et de nos pratiques, de comprendre à travers différents écrits l'évolution des réflexions autour de ce thème et d'en tirer des enseignements sur différentes manières de mettre en scène les sciences.

Dans un grand nombre de cas, la thématique méditerranéenne est présente et apporte une dimension signifiante supplémentaire (La Durance, Darius Milhaud, le Corail rouge...).

Pour finir, nous nous sommes également référés à d'autres œuvres, généralement connus, qui nous semblaient complémentaires de celles que nous avons nous-mêmes conçues et fabriquées.

Sur dix années de travail et de prise de recul, il y a ici un mouvement réflexif approfondi qui s'applique à une diversité d'œuvres qui constituent, dans leur pluralité, un ensemble caractéristique et typique.

Si l'on procède à une observation et une première analyse de l'ensemble de ses réalisations, il est possible de discriminer:

- Une mise en scène didactique à visée vulgarisatrice

- Une mise en scène codifiée publicitaire, clip, magazine, voire fictionnelle

- Une mise en scène qui favorise la participation active du spectateur (et la construction d'un regard sur les sciences)

Si cette typologie peut sembler surprenante, en particulier par son apparente non homogénéité, il n'en est rien. Il y a bien là une organisation

Congrès national de la SFSIC, il sélectionne un corpus cohérent, d'émissions d'information sur le cerveau, pour pouvoir analyser l'évolution du discours télévisuel sur la vulgarisation scientifique. 
logique qui va de l'espace d'interprétation le plus contraint vers une perspective plus ouverte qui laisse de la place au spectateur et à la coconstruction des significations.

\section{La mise en scène didactique à visée vulgarisatrice ${ }^{5}$}

La mise en scène didactique qui tente de vulgariser renvoie à la construction d'une démarche rationnelle pour l'esprit. Elle s'appuie sur une progression régulière de la difficulté et de l'utilisation des concepts. Elle est logique, articulée, tente de mettre en perspective des enchaînements causaux de la pensée.

Cette mise en scène s'adapte bien à un cours, une expérience in vivo, une démonstration, un sujet traité comme un documentaire à vocation pédagogique.

Un principe élémentaire semble souvent appliqué, et comme dans l'apprentissage classique, le propos commence par ce qui est relativement facile du point de vue de « l'apprenant » pour passer ensuite, peu à peu, à des concepts ou des définitions plus ardus.

Cela passe par un effort particulier de la formulation (supérieur à celui que I'on peut déployer pour les spécialistes), mais aussi de l'organisation et de l'enchaînement des représentations utilisées. Pour vulgariser, il est souvent question de simplifier, de se limiter à quelques éléments clés et d'utiliser le moins de mots techniques. Il faut également reconnaître qu'à l'origine, être didactique semblait également vouloir dire emprunter les caractéristiques et les attributs du savant qui s'exprime face à des ignorants ${ }^{6}$ ou simplement du professeur qui s'adresse à sa classe.

Dans ce cas, la logique de progression du contenu est tellement importante, que c'est le texte lu qui, souvent, porte l'avancée méthodique des

${ }^{5}$ En 1961, dans son bulletin $N^{\circ} 1$, l'Institut de Cinématographie Scientifique distingue :

- Les films de recherche

- Les films de vulgarisation scientifique

- Les films d'enseignement

${ }^{6}$ Pour Yves Coppens, "vous vulgarisez à partir du moment où vous ne vous adressez pas à vos pairs".

Interview de Yves Coppens, le 12 février 2007, http://lexnews.free.fr/sciences.htm\#coppens

Consulté le 18 janvier 2011 
significations. La voix devient le support du savoir et de l'autorité. Comme à l'école, elle contient l'essentiel des informations du propos pédagogique. Le ton de la parole qui sied à l'exercice est souvent monocorde, manque d'intonations et d'émotion. L'image n'est là que pour être commentée, mais interagit finalement peu avec le son. L'un et l'autre n'établissent pas de dialogue et ne se donne pas "la parole » à tour de rôle. Le son pourrait parfois s'écouter seul, il n'y aurait pas beaucoup de différence.

Depuis près de vingt ans maintenant, cette approche vulgarisatrice, dont le contenu exploite majoritairement les résultats de la science, comme les théories ou les concepts, et rend souvent compte des principes comme s'il s'agissait d'une réalité définitivement acquise, figée et immuable, est à juste titre critiquée. D'une part, elle ampute la science d'une dimension essentielle qui la relie à la société. D'autre part, sa vocation purement pédagogique la rend souvent assez peu intéressante pour le spectateur, voire franchement ennuyeuse si le propos est clos au lieu d'être ouvert vers des incertitudes et si le ton est docte, au lieu d'être vivant, voire narratif.

Il y a, c'est vrai, une dimension rébarbative lorsque un objectif exclusivement didactique prévaut. La forme et la construction audiovisuelle sont alors soumises à une logique exclusivement centrée sur une acquisition formatée du savoir directement héritée des enseignements traditionnels. Dans certains cas, était-ce réellement nécessaire de faire un film! Les films écrits par les pédagogues non aguerris aux pratiques et aux langages audiovisuels sont peut-être en ligne de mire... ${ }^{7}$

Néanmoins il faut être prudent, certaines réalisations comme ce film d'animation de deux heures "Dimension, une promenade mathématiques " 8 , découpé en neuf parties, accessible sur Internet, est loin d'être soporifique, tout en restant centré sur la didactique la plus conventionnelle. Le ton employé, pourtant sérieux, est si clair. Le travail des images de synthèse anime le propos. Le texte, qui a chacun fois se place dans la peau d'un grand découvreur ou chercheur. Le juste temps laissé aux images et aux sons pour que chacun puisse saisir la progression des idées. Ces choix conduisent cette réalisation

\footnotetext{
7 Dans son ouvrage "L'école devant les écrans ", Geneviève Jacquinot analyse les rapports qui existent entre le mode de l'audiovisuel et celui de l'éducation.

${ }^{8}$ http://www.dimensions-math.org/
} 
à atteindre ses objectifs et à en faire indéniablement une œuvre intéressante et généreuse.

Ainsi, de façon paradoxale, l'audiovisuel didactique ne relève pas toujours du plus simple et surtout du plus simpliste. Par ailleurs, il se constitue, dans l'appropriation des outils numériques par les chercheurs, un continent audiovisuel encore mal exploré, qui va de formes minimales (le cours filmé) à des formes sophistiquées, utilisant par exemple l'image de synthèse 3D.

Nous avons pu mettre ce phénomène en évidence lors de l'expérimentation / réalisation du DVD «La Durance, parcours et regards ", en introduisant dans le DVD de courtes séquences utilisant les Systèmes d'Information Géographique ainsi que des outils informatiques d'analyse spatiale. L'objectif était de formuler des données scientifiques sur des paysages (lieux « clés » de l'univers durancien), travail relevant de la compétence du géographe, mais croisant et superposant les approches de plusieurs disciplines: histoire, géologie, démographie, climatologie, sociologie... Comment rendre compte de la complexité de ce « mille-feuilles » cognitif en une minute et 30 secondes ?

Partant de la notion existante de "géoramas»`nous avons ajouté celle de l'image animée pour élaborer celle de «géocinéramas».

En quoi une image de synthèse, donnant du relief à une carte topographique, apporte t-elle le sentiment d'être plus «précise», qu'un analogon audiovisuel ? Comme si l'image, étant débarrassée de la question du présent de sa captation et de ses contingences (la cartographie 3D n'a pas de lumière naturelle ou artificielle, n'offre pas d'indices de temporalité du type "petit matin brumeux» ou "coucher de soleil», n'a pas d'univers sonore...), et limitée par l'échelle de la carte, suggérait une vison «objective» des paysages.

Quelques années avant que "google earth» - repris par les animations des bulletins météo de la télévision - ne nous habitue à ces survols virtuels, nous avons expérimenté des déplacements par la méthode de calculs selon la courbe de Béziers.

${ }^{9}$ Géorama (du grec gè, terre, orama, spectacle), en 1823, un certain Delanglard imagina une sphère de plus de $30 \mathrm{~m}$ de circonférence, en toile vernissée, sur laquelle étaient représentées toutes les terres du globe. Placé au centre, le spectateur pouvait ainsi tout embrasser d'un seul coup d'œil, (alors qu'avec de grands globes, vus de l'extérieur, on n'aperçoit à la fois qu'une petite portion de la Terre). Géorama désigne également une représentation en relief, sur une échelle plus ou moins grande, de l'ensemble ou d'une partie de la Terre. 
L'animation de la cartographie 3D a révélé ses étonnantes possibilités en terme de point de vue. II semble en effet peu probable, même en mobilisant des ressources technologiques considérables (caméras spéciales de prises de vue aérienne, hélicoptères), de produire l'effet obtenu par un survol virtuel, à l'altitude et la vitesse souhaitée ${ }^{10}$. Mais cet effet n'a aucun intérêt (en dehors de la curiosité technique), s'il n'est accompagné d'une lecture de la carte.

C'est donc dans le rapport dialogique entre le commentaire et le déplacement que tout se joue. La pratique de l'analyse spatiale induit que l'image accrédite le commentaire qui, dans le même temps, fait exister l'image. L'exercice requiert une certaine habileté, et repose sur la maîtrise de plusieurs paramètres, dont les principaux sont la vitesse et l'altitude, appliquées aux panoramiques et aux travellings.

La possibilité de combiner un travelling vertical (une "descente») avec un panoramique circulaire $\left(360^{\circ}\right)$ multiplie les possibilités de l'analyse spatiale. Situer un lieu particulier en plan large ( exemple: la "clue» de Sisteron, passage étroit de la Durance au sein d'une ligne de fracture de la chaîne alpine), pour s'en approcher en travelling (combinaison vertical / horizontal), avant de le décrire à très basse altitude et à faible vitesse (travelling latéral + panoramique), permet de synthétiser et de visualiser les processus de formation d'un paysage.

Ainsi, de la géologie à l'action de l'homme dans l'histoire (position de la ville, situation militaire, territoires agricoles), l'espace strié montre ses plis et ses strates. Pour obtenir ce résultat, il faudra que la simulation de l'espace durancien dans ces "géocinéramas " obéisse au principe fondamental qui régit la création des images numériques « une fois que le créateur de l'image a défini l'objet à simuler, qui peut être une pomme ou un roman, il doit gérer l'accès visuel du regardeur à l'objet, bref son devenir d'image !» ${ }^{11}$.

Cette rapide description permet de souligner à quel point la complexité de l'espace filmé a orienté le choix de son intelligibilité vers un dispositif de réception censé reproduire celle-ci, ou du moins donner à l'utilisateur les moyens de (re)fabriquer une complexité par une «offre» de lecture plurielle. La lecture comparative et croisée des films est censée proposer la construction d'une représentation spatiale autonome.

\footnotetext{
10 Voir Couchot Edmond, Images, De l'optique au numérique. Hermes, Paris, 1988, p.181 et suivantes.

${ }^{11}$ Couchot Edmond, op. cité, p. 193.
} 


\section{Une mise en scène codifiée publicitaire, clip, magazine ou même fictionnelle}

Il existe un autre travail de mise en image et en son qui consiste à s'appuyer sur les codes du cinéma, de la télévision et plus généralement de l'audiovisuel. Ainsi, certains films, pour parler de la science, préfèrent prendre une forme publicitaire, celle d'un clip ou d'un magazine télévisuel ou alors emprunter aux formes narratives de la fiction.

Ces différents codes ne sont d'ailleurs pas à classer de la même façon.

Dans le cas d'un publicité ou d'un clip, le mode de lecture convoqué par le spectateur et les mondes possibles qu'il va pouvoir construire au regard des films interprétés ne peut le conduire à avoir une vision complexe de la science ou de la recherche. Il est cantonné à de belles images, un montage dynamique et rythmé, un commentaire usant de phrases courtes, des enchaînements élégants et stylés, mais il reste à la surface des choses et ne peut construire son propre discours.

Roger Odin - dans son approche de la sémio-pragmatique - identifie ainsi différents modes de lecture mobilisés par le spectateur en fonction de la situation de réception dans laquelle il se trouve et du message auquel il est soumis. Pour étayer notre propos, pour la pub ou le clip, les modes de lecture adoptés seront majoritairement moins centrés sur le discours (mode argumentatif / persuasif), la compréhension d'un sujet, la construction d'un monde complexe que sur des constructions s'intéressant au travail de production des images et des sons, renvoyant à l'esthétique, au rythme perçu ou même au côté spectaculaire présent dans les images ${ }^{12}$ et les sons. ${ }^{13}$

Faire une mise en scène codifiée clip ou publicité, c'est majoritairement aiguiller le spectateur sur un mode de lecture qui le laisse à la surface des

12 De nombreux laboratoires de recherche - en particulier en biologie, en physique, en chimie... - ont capté et stocké des images tout à fait spectaculaires des différentes expériences qu'ils ont menées.

${ }^{13}$ Parmi les modes mobilisés par Roger Odin, sont identifiés en particulier :

- Mode esthétique : voir un film en s'intéressant au travail des images et des sons

- Mode spectaculaire : voir un film comme un spectacle (de cirque par exemple).

- Mode énergétique : voir un film pour vibrer au rythme des images et des sons.

- Mode artistique : voir un film comme la production d'un auteur 
choses, en privilégiant le forme et non le fond, en ne favorisant pas une représentation de la science, mais plutôt en la faisant voir de façon à ce qu'elle apparaisse d'abord comme efficace, attrayante, dynamique, séduisante... II s'agit de la vendre plus que de la faire comprendre. La science n'est pas expliquée, le film qui la montre, l'expose, use de clichés ou de stéréotypes pour qu'elle soit rapidement associée aux qualités supposées ${ }^{14}$ qu'elle pourraient avoir.

II n'y a pas ou peu de réels contenus scientifiques et encore moins d'intention de lui donner une portée sociétale. Le propos sur la science s'arrête aux éléments «visibles ". II n'y a pas appropriation de connaissances par le spectateur, mais plutôt reconnaissance, par habitude ${ }^{15}$, d'une forme filmique codifiée qui réduit le champ des interprétations possibles, fait appel à un interprétant communément partagé et ne laisse pas de place à une appropriation du contenu.

On peut, à bien des égards, considérer les articles de Bruno Latour, "Le résistible objet des films scientifiques " ou "Comment traduire la science ? En la trahissant... ${ }^{16}$, rédigés avec le club Scientification, comme fondateurs d'une réflexion sur les films de science. Dans le premier, en particulier, il expose un point de vue engagé et écrit : "Pour éviter la création du vidéo-clip scientifique - monstre affreux né du croisement d'un pédagogue qui a honte de l'être et d'un cinéaste branché horrifié par les contenus qu'il doit couvrir - il est capital que les deux positions, celle du réalisateur et celle du chercheur se modifient à la fois. $\gg 17$

Dans cet article, ils, les auteurs du club Scientification, déplorent que les films scientifiques français soient - de manière quasi pathologique - peu intéressants (abstraits, ennuyeux et peu télégéniques). Une des raisons est selon eux le manque de culture scientifique et l'absolue nécessité lorsqu'on aborde un film de science de parfaitement connaître le sujet. Ces difficultés conduisent souvent le réalisateur à faire un film esthétique ou au ton décalé ou de lui donner l'apparence d'une fiction a priori

\footnotetext{
14 Les qualités, au sens de Peirce.

${ }^{15}$ Peirce utilise le terme « d'interprétant logique final » pour exprimer la démarche

16 «Comment traduire la science ? En la trahissant... » in Education Permanente n॰93/94 pp.71-81
}

${ }^{17}$ Bruno Latour, le résistible objet des films scientifiques, écrit avec le club scientification, p4 
plus intéressante et accessible, alors que le contenu scientifique ou didactique n'est pas réellement traité ou en tout cas pas suffisamment sérieusement.

La piste de la fiction - loin d'être décriée - est par ailleurs soutenue par les auteurs qui défendent l'idée de personnaliser l'objet ou la chose qui est à l'origine du contenu scientifique et d'introduire une intrigue qui va permettre de distiller le contenu scientifique. Depuis cet article, écrit en 1989, cette démarche de rapprochement du film scientifique et de la fiction s'est généralisée et même popularisée. Le film l'Odyssée de l'espèce, réalisé par Jacques Malaterre et diffusé en 2003 en est un exemple retentissant ${ }^{18}$, même si - comme le rapporte Yves Coppens - il n'a pas plu aux scientifiques qui considèrent que seuls ce dont on a la preuve peut être exploité et qu'au delà, c'est de l'imagination. II rajoute : "Le chemin est étroit entre le respect de l'information scientifique et la volonté de vulgariser, comme il l'est entre le plaisir du spectacle et la vérité du document. »

La fiction est-elle alors finalement garante de l'intérêt du film ou serait-elle au contraire à l'origine des limites scientifiques du projet et nuirait donc à son intérêt ? L'imagination serait-elle à bannir dans les films scientifiques ?

Dans tous les cas, la question de la transmission des connaissances au plus large public se trouve posée. Mais, comme le souligne Gérard Leblanc : «Aussi précisément définis soient les publics visés, on observe des résistances, on constate des multiples échecs, résistances et échecs qu'on attribue d'ordinaire à une inadéquation dans l'élaboration formelle des messages à transmettre. II y aurait - chez les scientifiques qui se préoccupent de communication comme chez les cinéastes avec lesquels ils collaborent - une réflexion insuffisante sur la transformation du discours scientifique en message audiovisuel pertinent $\gg 19$. Ce constat trouve son prolongement dans le recours aux technologies numériques interactives, présentées par les commanditaires institutionnels et scientifiques - avec l'assentiment bienveillant des concepteurs et industriels des technologies concernées - comme la voie du salut : le public, grâce à des machines proposant des usages ludiques - la fameuse «interactivité »

\footnotetext{
18 Le 8 janvier 2003, la diffusion du l'Odyssée de l'espèce a réuni 8745000 téléspectateurs soit "la meilleure audience pour une première partie de soirée pour France 3 depuis la Coupe du monde de football en 1998, et un record historique pour la diffusion d'un documentaire sur la chaîne ».

19 Leblanc Gérard Scénarios de la Science, in Scénarios du réel, I'Harmattan, Paris 1997, p.168.
} 
accèderait à coup sûr aux joies de la connaissance scientifique et en deviendrait le partenaire assidu.

Or l'audiovisuel (y compris sous ses formes considérées comme les plus modernes), ne peut tenir lieu de méthodologie pour apprendre. Comprendre les difficultés, voire les impasses auxquelles peut aboutir ce type de projet suppose d'en observer les conditions de production, et répondre à la double question!: qui demande quoi et pour quels usages ? La pratique de la commémoration encouragée par les organismes internationaux et les institutions (en France, le phénomène est érigé en système), induit la mobilisation de ressources financières proposées aux télévisions comme à l'ensemble des producteurs de «biens culturels» (y compris ceux du cinéma). Cette politique de financement axée sur la commémoration d'un événement a sans aucun doute de bonnes raisons d'exister et relève de préoccupations vertueuses, mais n'est pas sans avoir des effets pervers sur la création ellemême. La recherche de financements étant au centre de la survie du secteur économique concerné, la démarche a priori naturelle consistant à proposer des programmes contre un financement est inversée. «Faites quelque chose sur... et vous aurez de l'argent» devient la règle. D'autre part, il n'est pas acquis, malgré la mise en place de comités d'experts réputés compétents, chargés d'évaluer et de décider de la qualité et de l'opportunité des projets, que les intentions soient toutes claires et les résultats nécessairement au niveau souhaité. Les experts ne sont pas, d'ailleurs, toujours soucieux de méthodologie: les représentants des institutions scolaires et culturelles s'inquiètent du public potentiel et ouvrent ainsi les portes au «ludique», voire au spectaculaire censé rassembler le plus grand nombre. Les représentants de la Recherche et de la Science ne sont pas de leur côté à l'abri de telles turpitudes. La médiatisation des hommes et des travaux de recherche est devenue une composante importante de l'avenir des laboratoires. La question de la commande est donc déterminante : elle oriente, dans un même mouvement, les stratégies d'écriture et de diffusion. Sur ce point l'expérimentation que nous avons menée dans le cadre de «l'Année mondiale de la physique» («Mécaniques relatives», 2005), les garanties d'un projet scientifique semblaient a priori réunies (rassemblement, au sein d'une Université, de laboratoires des Sciences de la Matière et d'une équipe de Recherche en audiovisuel) : encore fallait-il, pour obtenir des moyens de le mener à bien, en justifier la portée. Les experts des institutions culturelles et scolaires considéraient par avance et de façon catégorique la venue des chercheurs, ou la projection des films «dans les classes» comme un échec. Sans poser la question du pourquoi de cet échec, la demande devient donc 
celle de la détermination d'un autre espace de réception, incluant des objets audiovisuels de type "participatif», dans lesquels "le multimédia " et "l'interactif » tiennent lieu de discours. Exit la cristallisation artistique et politique des enjeux de la Science, au profit du champ des loisirs éducatifs ${ }^{20}$. II importe de déjouer des confusions. La mise en œuvre de technologies sophistiquées qui remettent en cause la linéarité du film et la démultiplication des points de vues ne signifie pas en soi que le spectateur pourra construire un regard pertinent sur le sujet. Si le dispositif ne crée aucun écart entre le déjà connu du spectateur et ce qu'il voit et entend, s'il est fondé sur des codes déjà éprouvés et des processus de reconnaissance de soi dans l'image et le son, il reste englué dans les représentations fictionnelles et publicitaires de la Science. Ce que "Mécaniques relatives» nous aura appris tient dans l'approfondissement de la question de l'espace de réception : aucune scénographie ne saurait faire l'économie d'une réflexion sur l'espace d'interaction entre le regard du filmeur et celui du "visiteur-découvreur», un espace de subjectivité où les deux regards se côtoient, se superposent, s'interrogent. Dans le domaine des sciences, la production des dispositifs visuels dépend étroitement des conditions (idéologiques, cognitives, technologiques) dans lesquelles elle s'inscrit. Cette production participe de la construction d'un regard sur la science, peut bouleverser des représentations (celles du corps, de la Terre, de l'univers, du vivant...) et élaborer une/des vison(s) du monde. La transformation et les mises en scène des énoncés (faits bruts) de la (des) science(s) en énoncés sociétaux (cristallisation politique et artistique des enjeux) ne peut échapper à la division du travail qui fait que dans nos sociétés la culture scientifique n'apparaît pas comme une nécessité vitale. Comment éviter (et contrer) le discours spectacle de la télévision sur les sciences, le discours «distractif», ou les dérives de la science-fiction?

Pour tenter d'apporter des pistes de réflexions, nous poursuivons notre logique de catégorisation.

\section{La mise en scène qui participe de la construction d'un regard sur les sciences.}

Une autre approche pour le film scientifique consiste, bien au-delà de la nécessité de suivre une didactique de la vulgarisation ou de donner au film des

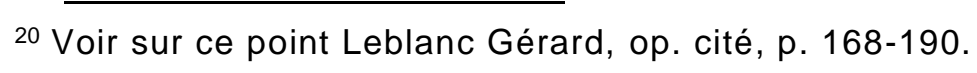


apparences fictionnelles, à proposer un contenu à investir, un film qui implique le spectateur et lui offre à construire son propre regard sur les sciences.

Comme l'écrit Bruno Latour, "II est important de présenter les sciences comme proches et intéressantes, comme accessibles (mais pas comme simpliste). De parvenir à les dramatiser, à les insérer dans un cadre qui les dynamise. Elles ne sont pas un savoir à absorber, mais une situation à comprendre et à saisir dans son évolution et son rapport au monde. "

Pour Francis Gast, il ne s'agit plus nécessairement d'introduire de la fiction dans le film scientifique, mais seulement d'utiliser le potentiel narratif de la science c'est-à-dire de raconter la science. Aborder la science ainsi, c'est éviter l'écueil du professeur caché au profit, précisément, du conteur ${ }^{21}$.

Narrer la science ne signifie pas lui adjoindre nécessairement une dimension fictive et la transformer en un récit imaginaire. Non, il s'agit avant tout de ne pas la décrire en oubliant qu'elle contient tous les éléments qui peuvent lui faire prendre la forme d'une histoire qui se construit. Parler de la science, mais aussi de la recherche, c'est rendre compte d'un point essentiel : sa progression, sa lente avancée, mais c'est aussi la rendre humaine, manifester ses doutes, ses incertitudes, ses questionnements. II s'agit avant tout de raconter comment la science est vivante et faillible.

Narrer la science, c'est utiliser l'univers concret et quotidien de la science et non seulement rendre compte des résultats et concepts figés qu'elle génère. Cet univers, ce sont les appareils de mesure, les humains qui les utilisent, les lieux et les espaces réels, les relations entre chercheurs, l'organisation du travail... Bruno Latour utilise une formule qui résume l'essentiel, c'est la science «telle qu'elle se fait».

D'une part la présenter de cette façon c'est la présenter telle qu'elle est et d'autre part cela autorise le spectateur à investir le film pour y construire ses propres hypothèses, y raccrocher sa propre éthique, ses propres préoccupations, et de manière plus concrète encore s'interroger sur le métier de scientifique, les enjeux de la science et les débats qui y sont associés.

\footnotetext{
${ }^{21}$ Francis Gast, Filmer la science, La lettre de la Safire (Société des auteurs réalisateurs de films indépendants en Région Est), Mai 2000, pp. 19-20
}

http://umb-www-01.u-strasbg.fr/arts/documents/pdf_jour_etud/SAFIRE.pdf 
Au sens codifié, aux propos scientifiques qui s'appuient sur des assertions, aux contenus scientifiques qui mettent le spectateur à distance, aux images et aux sons qui se donnent pour être interprétés en surface, on substitue une narration qui dynamise, une structure qui questionne, des articulations qui sont à construire, des événements en mouvement qui ne sont pas stabilisés, un contenu qui peut interagir avec des questionnements du quotidien...

La question des "dispositifs Arts / Sciences " se pose à ce niveau. Lorsque les mises en scène contemporaines de la science sont directement liées à des projets de recherche, activités artistiques et scientifiques se nourrissent l'une de l'autre. Installations, multi écrans, parcours et paysages sonores, méta montage, projections monumentales, systèmes de géolocalisation ou d'information géographique, interactions à distance, (nomadisme audiovisuel) constituent des énoncés matériels déstabilisateurs du spectateur, à l'articulation de l'observation et de l'imaginaire, du visible et de l'invisible. II s'agit de confronter ce qui pourrait être avec ce qui est. C'est ce que nous avons développé avec les physiciens ("Mécaniques relatives", les spécialistes de la biodiversité marine ("Rêves de Corail », les astrophycisciens ("La vie ailleurs»).

Les écritures de ces scénographies sont au centre de la démarche, incluant la question des dispositifs de diffusion : le contexte d'usage possède des effets configurants sur les formes, leurs valeurs et leurs agencements.

Cette mise en scène peut bouleverser des représentations et élaborer des visions du monde.

Les écritures (documentaire, fiction, expérimental) de ces mises en scène sont au centre de la démarche, englobant la question des dispositifs de diffusion.

Ces pistes de travail et de recherche ont été élaborées notamment dans le cadre d'une Expérimentation audiovisuelle, Rêves de corail, menée en collaboration avec les chercheurs du laboratoire DIMAR (Diversité, évolution et écologie fonctionnelle marine), sous la direction de Jean-Pierre Féral, Centre d'Océanographie Marine, CNRS). Objectif : un transfert de qualité des connaissances vers la société, présenter la biologie et la génétique du développement d'une espèce marine, poser clairement la question de la diversité génétique, des impacts des changements climatiques, des relations entre les milieux marins et terrestres. La découverte du Corail Rouge de Méditerranée, espèce en voie de disparition, se devait de tenir compte de la 
multiplicité des approches possibles : enjeux environnementaux, scientifiques, humains, passé, présent, avenir constituaient autant de point de vues entrecroisés, sans oublier la part de l'imaginaire construite sur la mythologie et l'histoire du Corail Rouge en méditerranée.

L' installation audiovisuelle fut conçue comme un énoncé matériel dans lequel les images et leurs commentaires renvoyaient de façon quasi systématique à la dimension spatiale hybride de la mer, faite de formes agencées de façon variable, de représentations et d'images mentales fondées sur des lieux concrets et des structures matérielles. De par leur double signification, chaque image et son commentaire sont tour à tour concept et représentation.

L'image-concept emblématique de la plongée, devenue descente intérieure, puis cheminement dans l'installation, constitua l'aboutissement concret de cette démarche. Matérialisé par des repères de profondeur sur les parois le long du parcours, l'espace se distribue de part et d'autre d'une surface : la navigation devient double : horizontale et verticale, mêlant réel et imaginaire (analyse des fossiles de Corail rouge au carbone 14 et antiquité gréco-latine, de la plante animal aux Gorgones et à Persée...). Il faut ici souligner - point de départ d'un champ de recherche associé à celui de la mise en scène des sciences - en quoi le rôle du son, de la spatialisation sonore a joué dans le cas évoqué un rôle déterminant. Cette question, qui ne peut être ici abordée de façon exhaustive, doit être placée au centre de notre questionnement sur la place et l'investissement du visiteur-spectateur et de son implication dans la proposition qui lui est faite.

\section{Conclusion}

Retenons donc en vue de développements ultérieurs une classification des formes de mises en scènes audiovisuelles scientifiques:

- La mise en scène didactique qui tente d'expliquer, de vulgariser un principe scientifique considéré comme complexe et qui place le spectateur dans la position de celui qui doit apprendre un énoncé.

- La mise en scène publicitaire qui met en valeur, un laboratoire, un centre de recherche public ou privé, une université ou même un chercheur, qui traite des informations dans un sens spectaculaire, applique des schémas stéréotypés à une lecture de la science non-réelle.

- Les mises en scènes qui impliquent le spectateur, le déstabilisent, introduisent des passerelles entre réel / imaginaire, visible / non-visible... 
Sur ce dernier niveau de mise en scène, nous avons pu observer au cours de nos réalisations / expérimentations, combien la réalisation d'un film relève aussi d'un métier qui a ses contraintes et a besoin de ses espaces de liberté22.

Il est essentiel que le cinéaste et le scientifique trouvent à se comprendre, se fassent confiance et construisent une discours qui ne soit pas figé, mais présentent la science comme un processus, une création en cours, un work in progress.

Mais il semble essentiel, au-delà de cette catégorisation, d'en tirer des enseignements pour placer le film scientifique dans une dynamique nouvelle, une dynamique qui finalement ne lui donne pas une place à part. Le film scientifique, comme tout film documentaire ou de fiction, doit tenter d'amener le spectateur à saisir les contours d'un contenu, mais surtout doit l'inciter à s'interroger, à croiser les contenus du film avec des idées qui préexistaient en lui, à s'approprier une représentation personnelle de l'univers qu'il découvre, à repousser plus loin son questionnement...

Au regard des articles et des ouvrages que nous avons lus, il semble que l'objectif didactique ou de vulgarisation, tel qu'il existait il y a encore une dizaine d'années, ne puisse plus constituer un objectif louable tant il est absolument nécessaire d'introduire un regard éthique et sociétal sur la science. II n'est plus possible de s'intéresser au domaine scientifique sans s'ouvrir à ces questions fondamentales qui permettent de questionner la science et d'éviter que les résultats qu'elle obtient soient isolés du monde dans lequel nous vivons.

\section{Bibliographie}

BABOU, Igor, Histoire d'une confrontation. Le discours télévisuel à propos de la science, Actes du XII Congrès national de la SFSIC, du 10 au 13 janvier 2001

COUCHOT Edmond, Images, De l'optique au numérique. Hermès, Paris, 1988

EVERAERT-DESMEDT, Nicole, Le processus interprétatif, Introduction à la sémiotique de Charles Sanders Peirce, Liège, Mardaga,1990

22 Francis Gast, Ibid 
HAGUENAUER, Martine, Les films scientifiques et techniques français, BBF, 1962, $n^{\circ} 4, \mathrm{p} 207-218$

http://bbf.enssib.fr/consulter/bbf-1962-04-0207-002, consulté le 17 janvier 2011

JACQUINOT, Geneviève, L'école devant les écrans, Paris, ESF, 1985

LATOUR, Bruno, avec le Club Scientifiction, Le résistible objet des films scientifiques, analyse sémiotique, miméo, 50 p. (avec le Club Scientifiction), 1989

http://www.bruno-latour.fr/poparticles/poparticle/p029.html, consulté le 12 janvier 2011

LATOUR, Bruno, avec le Club Scientification, Comment traduire la science ? En la trahissant..., in Education Permanente, $n^{\circ} 93$, pp. 71-81, 1988

LEBLANC Gérard Scénarios de la Science, in Scénarios du réel, l'Harmattan, Paris 1997

ODIN, Roger, Cinéma et réception, collectif d'auteurs dirigé par Roger Odin et Jean-Pierre Esquenazi, Hermes, 2000

\section{Webliographie}

http://lexnews.free.fr/sciences.htm\#coppens, consulté le 16 janvier 2011 Interview de Yves Coppens du 12 février 2007

http://www.mif-sciences.net/francais/mag/siecle/filmdoc-alloul.htm, consulté le 15 janvier 2011

1889-1989 : Un siècle de cinéma scientifique en France

http://umb-www-01.u-strasbg.fr/arts/documents/pdf_jour_etud/SAFIRE.pdf, consulté le 9 janvier 2011

GAST Francis, Filmer la science, La lettre de la Safire (Société des auteurs réalisateurs de films indépendants en Région Est), Mai 2000, pp. 19-20 